\title{
Experimental and theoretical study of the energy loss of Be and B ions in $\mathrm{Zn}$
}

\author{
E. D. Cantero, ${ }^{1}$ R. C. Fadanelli, ${ }^{2}$ C. C. Montanari, ${ }^{3,4}$ M. Behar, ${ }^{2}$ J. C. Eckardt, ${ }^{1}$ G. H. Lantschner, ${ }^{1}$ \\ J. E. Miraglia, ${ }^{3,4}$ and N. R. Arista ${ }^{1}$ \\ ${ }^{1}$ Centro Atómico Bariloche and Instituto Balseiro, Comisión Nacional de Energía Atómica, 8400 S.C. de Bariloche, Argentina \\ ${ }^{2}$ Instituto de Física, Universidade Federal do Rio Grande do Sul, Avenida Bento Goncalves 9500, 91501-970, Porto Alegre, RS, Brazil \\ ${ }^{3}$ Instituto de Astronomía y Física del Espacio, Casilla de Correo 67, Sucursal 28, 1428 Buenos Aires, Argentina \\ ${ }^{4}$ Departamento de Física, Facultad de Ciencias Exactas y Naturales, Universidad de Buenos Aires, 1428 Buenos Aires, Argentina
}

(Received 18 December 2008; revised manuscript received 8 March 2009; published 23 April 2009)

\begin{abstract}
Energy-loss measurements and theoretical calculations for $\mathrm{Be}$ and $\mathrm{B}$ ions in $\mathrm{Zn}$ are presented. The experimental ion energies range from $40 \mathrm{keV} / \mathrm{u}$ to $1 \mathrm{MeV} / \mathrm{u}$, which includes the energy-loss maximum and covers a lack of experimental data for these systems from intermediate to high energies. The measurements were performed using the Rutherford backscattering technique. The ab initio calculations are based on the extended Friedel sum rule-transport cross-section method for the valence electrons and the Shellwise local plasma approximation for the bound electrons. A comparison of these calculations to the present experimental data for $\mathrm{Be}$ and $\mathrm{B}$ and previous values for $\mathrm{H}, \mathrm{He}$, and $\mathrm{Li}$ ions on the same target is included. This confirms the applicability of the employed theoretical framework also for ions of intermediate atomic number.
\end{abstract}

DOI: 10.1103/PhysRevA.79.042904

PACS number(s): $34.50 . \mathrm{Bw}$

\section{INTRODUCTION}

The study of energy loss of swift ions in zinc is a subject of interest in both basic science and technological applications such as plating or multilayer system composed by thin $\mathrm{Zn} / \mathrm{Te}$ used to build electronic devices. However, with the exception of different studies of stopping of protons in $\mathrm{Zn}$, the experimental information is scarce [1]. This feature is basically due to difficulties in the preparation of thin $\mathrm{Zn}$ samples by evaporation because of its condensation properties.

In the last few years the stopping powers of $\mathrm{He}$ [2] and $\mathrm{Li}$ [3] ions in $\mathrm{Zn}$ have been measured in a wide energy range and the results were compared with $a b$ initio theoretical calculations. A good theoretical-experimental agreement was obtained not only with our own $\mathrm{Li}$ and $\mathrm{He}$ data but also for previously published stopping-power data for $\mathrm{H}$ and $\mathrm{He}$ ions $[4,5]$.

Concerning Be and $\mathrm{B}$ ions in $\mathrm{Zn}$, there is only one set of measurements for each ion, which covers a small energy range [6]. On the other hand, there are semiempirical predictions by the SRIM code [7], but not first-principles calculations.

The objective of this work is to provide stopping-power data for $\mathrm{Be}$ and $\mathrm{B}$ on $\mathrm{Zn}$ in a wide energy interval and to extend the $a b$ initio calculations already used for $\mathrm{H}, \mathrm{He}$, and $\mathrm{Li}$ [3] in order to test the theoretical scheme for heavier projectiles. This new test of the theoretical approach is important since the theoretical scheme includes a combination of perturbative and nonperturbative contributions which vary in different ways when the strength of the interaction increases due to the higher ionic charges.

The measurements were performed on $\mathrm{Zn}$ thin films using the Rutherford backscattering (RBS) technique. The ab initio theoretical description of the stopping power considers the valence electron contribution in the binary collisions framework using the nonperturbative quantum approach based on the extended Friedel sum rule-transport cross-section
(EFSR-TCS) scheme [8-10] and the core electron contribution in the perturbative approximation of an inhomogeneous free-electron gas [11-15] within the shellwise local plasma approximation (SLPA) [16].

The experimental procedure is described in Sec. II. The data analysis is presented in Sec. III, followed by a description of the theoretical formulation in Sec. IV. The experimental and theoretical results are discussed in Sec. V and compared to the semiempirical predictions of the SRIM code [7].

\section{EXPERIMENTAL PROCEDURE}

The energy loss for Be and B in $\mathrm{Zn}$ was determined by the same technique as in our previous work [3] that is using the RBS technique, at the $3 \mathrm{MV}$ Tandetron of the Instituto de Fisica da Universidade Federal do Rio Grande do Sul (IFUFRGS), Brazil. The energy range covered by the present measurements varies between $800 \mathrm{keV}$ and $10 \mathrm{MeV}$.

As in the cited work [3], Au/Zn/Au multilayer target films deposited on Si wafers were employed. Due to the wide energy range measured in this experiment different foils were used, the thicknesses being the following: (38 \pm 3$)$, $(138 \pm 7)$, and $(210 \pm 11) \mathrm{nm}$. These values were determined by energy-loss measurements using proton beams. The corresponding stopping power of $\mathrm{Zn}$ for protons was taken from Refs. [17,18].

The sample was mounted on a four-axis goniometer. For each energy the angle between the normal to the sample and the beam was varied between $0^{\circ}$ and $60^{\circ}$ leaving the detector position fixed. In Fig. 1 a scheme of the experimental geometry and a typical RBS spectrum resulting from the $138 \mathrm{~nm}$ multilayer target taken with a ${ }^{9} \mathrm{Be}$ beam at $4 \mathrm{MeV}$ are shown. It can be clearly observed that the two peaks of the Au markers and the main peak corresponding to $\mathrm{Zn}$ are well separated.

In the present case the combined detector and electronic resolution was of the order of $15 \mathrm{keV}$. The selection of the 


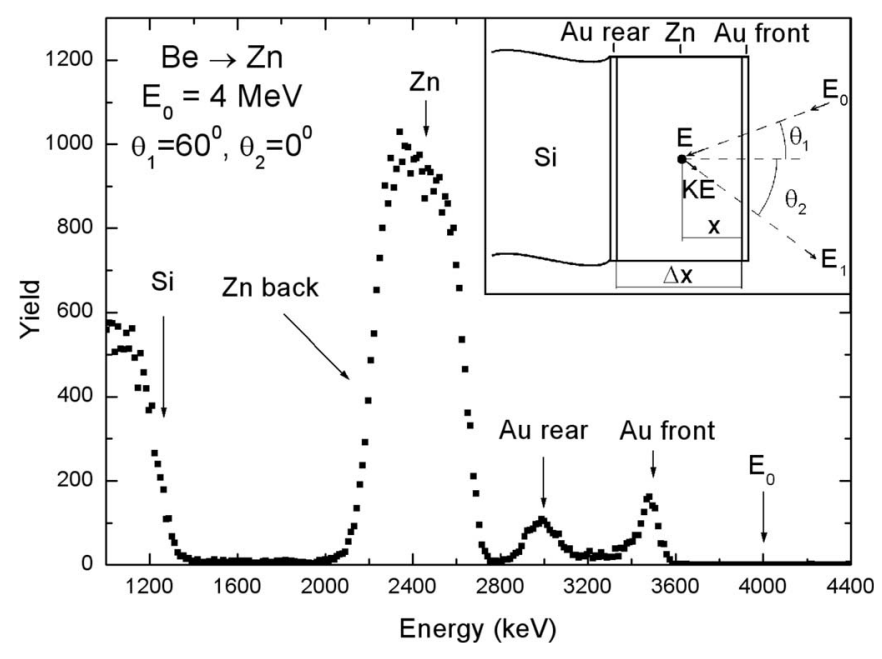

FIG. 1. ${ }^{9} \mathrm{Be}$ RBS spectrum of a $\mathrm{Au} / \mathrm{Zn} / \mathrm{Au}$ system taken at 4 $\mathrm{MeV}$. Inset: scheme of the backscattering geometry.

sample thicknesses was done according to the energy of the beam.

The ${ }^{16} \mathrm{O}(\alpha, \alpha){ }^{16} \mathrm{O}$ reaction at $E=3035 \mathrm{keV}$ was used in order to check the $\mathrm{O}$ content in the samples. In all the cases it was determined that the present $\mathrm{O}$ concentration was less than $3 \%$. In addition, in order to minimize the ion beam induced damage on the sample, for each energy we have used a fresh spot by shifting its position with the fourth axis of the goniometer.

\section{DATA ANALYSIS}

The data analysis was performed by a procedure based on that described in [3], modified to take into account the energy loss along the incoming and outgoing paths. This feature is relevant in the present case where heavier ions, i.e., larger energy losses, are considered.

The energy loss $\Delta E$ of the $\mathrm{Be}$ and $\mathrm{B}$ ions in the $\mathrm{Zn}$ layer of thickness $\Delta x$ was evaluated by determining the position of the edges of the corresponding energy distribution. Since the maximum energy transferred in a single process is much smaller than the above-mentioned energy-loss straggling, following Bohr criterion [19], the energy-loss distribution has a Gaussian shape. As the experimental resolution is also Gaussian-type, the back edge of the energy distribution can be fitted with the error function and the leading edge with the complementary error function.

The stopping power $d E / d x$ can be obtained from the experimental data for the ions backscattered at a depth $x$ of the film through the following relation based on the mean energy approximation [20]:

$$
\Delta E(x)=\left.\frac{x K}{\cos \theta_{1}} \frac{d E}{d x}\right|_{\bar{E}_{\text {in }}}+\left.\frac{x}{\cos \theta_{2}} \frac{d E}{d x}\right|_{\bar{E}_{\text {out }}},
$$

where $K$ is the kinematic factor, $\theta_{1}$ and $\theta_{2}$ are the angles of the sample normal with the incoming beam and the detector position, respectively, and $d E /\left.d x\right|_{E_{i}}$ is the stopping power of $\mathrm{Zn}$ for the Be or B ions of energy $E_{i}$.
In the mean energy approximation it is assumed that the energies along the inward $\left(\bar{E}_{\text {in }}\right)$ and outward $\left(\bar{E}_{\text {out }}\right)$ paths are

$$
\begin{gathered}
\bar{E}_{\text {in }}=\frac{1}{2}\left(E+E_{0}\right), \\
\bar{E}_{\text {out }}=\frac{1}{2}\left(E_{1}+K E\right),
\end{gathered}
$$

where $E_{0}$ is the energy of the incident particles, $E$ is the energy immediately before scattering at a depth $x$, and $E_{1}$ is the energy of the backscattered particles emerging from the surface. For each spectrum, $E$ was determined using the energy-loss ratio method as described in Ref. [20].

Considering Eq. (1) for ions backscattered at the back of the $\mathrm{Zn}$ film, $x$ equals the film thickness $\Delta x$. When measuring at two (or more) different geometries, a system of equations is obtained, which can be solved to get the stopping-power values $\left.\frac{d E}{d X}\right|_{E_{\text {in }}}$ and $\left.\frac{d E}{d X}\right|_{E_{\text {out }}}$. For each energy $E_{0}$ four measurements were performed under different geometrical conditions $\left(\theta_{1}=0^{\circ}, 20^{\circ}, 40^{\circ}\right.$, and $60^{\circ}$ and with $\left.\theta_{2}=60^{\circ}-\theta_{1}\right)$. The stopping power and their corresponding energies $\overline{E_{\text {in }}}$ and $\overline{E_{\text {out }}}$ were taken as the mean values of the results.

Proceeding in the same way for every energy, the stopping-power curves for $\mathrm{Be}$ and $\mathrm{B}$ in $\mathrm{Zn}$ were obtained. These data are based on the thicknesses determined using the proton stopping power chosen in the present case $[17,18]$. If other databases are used, the present results should be accordingly adjusted.

\section{THEORETICAL CALCULATIONS}

The theoretical scheme to calculate the energy loss follows the one described in Ref. [3]. In the energy range of the present calculations $(10-10000 \mathrm{keV} / \mathrm{u})$ this energy loss is due to the inelastic stopping by the target electrons, except at the lowest energies where the elastic scattering with the atomic cores varies from $15 \%$ at $10 \mathrm{keV} / \mathrm{u}$ down to $2 \%$ at $40 \mathrm{keV} / \mathrm{u}$ to become insignificant at higher energies [7].

The total stopping cross section $S$ is the sum of the partial-stopping terms $S_{q}$ corresponding to each projectile with charge state $q$ weighted with the fraction of charge $\phi_{q}(v)[21]$, namely,

$$
S(v)=\sum_{q=0}^{Z_{P}} \phi_{q}(v) S_{q}(v) .
$$

The partial-stopping cross sections $S_{q}$ are determined by the interaction of the dressed ion (with $N=Z_{P}-q$ bound electrons) and all the target electrons. In this calculation the small contribution [3] of the projectile electron excitation, commonly referred to as antiscreening contribution, will not be considered.

The $S_{q}$ values are calculated as the sum of the contributions due to the interaction between the projectile and the outer electrons and the interaction with the different target bound shells as described in our previous work [3]. The atomic structure of the projectile is represented by the Fourier terms 


$$
\rho_{q}(k)=Z_{P}-\sum_{n=1}^{N}\left\langle\varphi_{n}\left|e^{i \mathbf{k} \cdot \mathbf{r}}\right| \varphi_{n}\right\rangle,
$$

which may be interpreted as a kind of effective charge of the projectile screened by the $N$ bound electrons. This $\rho_{q}(k)$ may be expressed analytically in terms of the Slater-type expansions for the atom or ion [22]. In the case of Be and B ions, $\rho_{q}(k)$ is given by

$$
\rho_{q}(k)=Z_{P}-N_{1 s} Z_{1 s}(k)-N_{2 s} Z_{2 s}(k)-N_{2 p} Z_{2 p}(k),
$$

with $N_{1 s}, N_{2 s}$, and $N_{2 p}$ being the numbers of electrons bound in these shells, if any. Simple expressions for the screening functions $Z_{1 s}(k)$ and $Z_{2 s}(k)$ were given earlier [3]; the new term that appears for B atoms is

$$
Z_{2 p}(k)=\left[1-X_{2 p}(k)\right] /\left[1+X_{2 p}(k)\right]^{4},
$$

with $X_{2 p}(k)=k^{2} /\left(-2 n^{2} \varepsilon_{2 p}\right)$, with $\varepsilon_{2 p}$ being the binding energy of the $2 p$ shell.

The total stopping cross section for $\mathrm{Be}^{+q}\left(\right.$ or $\left.\mathrm{B}^{+q}\right)$ can be expressed as

$$
S_{q}=S_{q, \text { valence }}+\sum_{n \ell} S_{q, n \ell},
$$

where $S_{q \text {,valence }}$ represents the interaction between the projectile and the target valence electrons, while the $S_{q, n \ell}$ terms yield the contribution of each individual shell of the target atoms. In the present approach, the contributions of the outer and inner electrons of $\mathrm{Zn}$ are calculated independently. For the outer or valence electrons, the EFSR-TCS for a gas of electrons with $r_{s}=2.02$ is employed, corresponding to three electrons per atom of $\mathrm{Zn}$. This stems from the experimental plasmon energy of $17 \mathrm{eV}$ [23] which indicates the contribution, on average, of one electron from the $3 d$ shell, together with the two electrons from the valence band. For the inner shells $\left(1 s^{2}\right.$ up to $\left.3 d^{9}\right)$ the calculation is performed by using the SLPA. Both models are summarized in the following sections.

\section{A. Extended Friedel sum rule-transport cross-section method}

This approach contains three basic steps. First, the screening of the ion by valence electrons is analyzed using scattering theory and performing numerical integrations of the Schrödinger equation to calculate the corresponding phase shifts; this procedure is repeated iteratively until the phaseshift values so obtained satisfy the extended Friedel sum rule, which is a general condition for screening of moving ions [8]. Once the self-consistency is achieved, the transport cross section is evaluated as a function of the relative electron-ion velocity. And finally, an integration over relative velocities is performed, considering an ion with velocity $v$ and a Fermi distribution of electron velocities in the target $[9,10]$. This approach is referred to as a nonlinear (or nonperturbative) calculation, which applies to all orders in the interaction potential and for wide range of velocities, while the dielectric-function method is based on a perturbative approximation and is therefore suitable for intermediate and high energies.

In Figs. 2 and 3 the EFSR-TCS results are displayed for the different charge states of the Be and $\mathrm{B}$ ions, respectively.

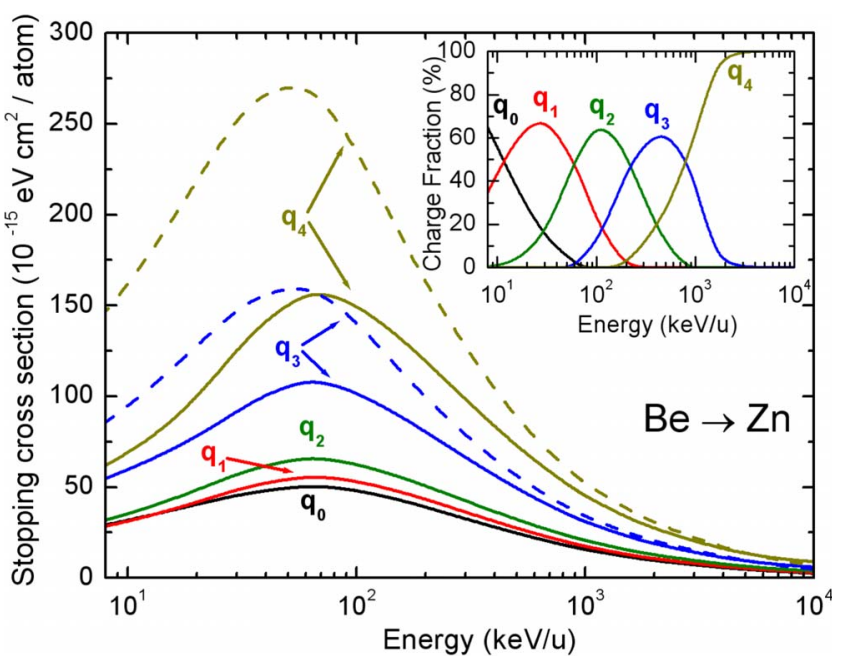

FIG. 2. (Color online) Valence electron contribution to the stopping cross section of $\mathrm{Be}^{+q}$ on $\mathrm{Zn}$. The curves correspond to $q$ $=0,1,2,3,4$ from down to top. Solid lines, nonperturbative EFSRTCS calculations [10]; dashed lines, perturbative results using Mermin dielectric function [24]. Inset: Percentage of each charge state of $\mathrm{Be}$ ions in $\mathrm{Zn}$ as a function of the impact energy, given by the semiempirical predictions of CASP [25].

These results are compared to those obtained by employing the dielectric formalism with Mermin dielectric functions [24], which hinges on perturbation model assumptions. The equilibrium charge states of $\mathrm{Be}$ and $\mathrm{B}$ in $\mathrm{Zn}$ depend on the impact velocity. For the present calculations, the semiempirical predictions of charge states obtained from the CASP code [25] are displayed in the figures as an inset.

The differences between the nonlinear (EFSR-TCS) results and the linear (dielectric) calculations, displayed in Figs. 2 and 3, are very large for the highest charge states. The

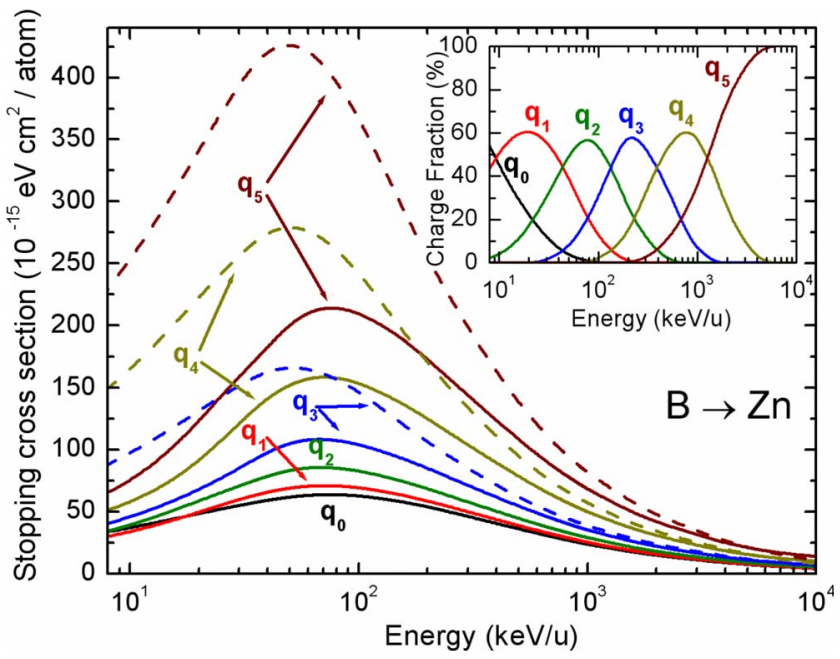

FIG. 3. (Color online) Valence electron contribution to the stopping cross section of $\mathrm{B}^{+q}$ on $\mathrm{Zn}$. The curves correspond to $q$ $=0,1,2,3,4,5$ from down to top. Solid lines, nonperturbative EFSR-TCS calculations [10]; dashed lines, perturbative results using Mermin dielectric function [24]. Inset: Percentage of each charge state of $\mathrm{B}$ ions in $\mathrm{Zn}$ as a function of the impact energy, given by the semiempirical predictions of CASP [25]. 
nonlinear effects are most significative at low and intermediate energies. The relative magnitude of these effects decreases for less charged projectiles (not shown in these figures).

\section{B. Shellwise local plasma approximation}

As indicated earlier, the contribution of target bound electrons is calculated by using SLPA $[3,16]$. This model considers the response of the bound electrons as that of a freeelectron gas of inhomogeneous density. It considers independent response of each $n l$ shell of target electrons. In this approach a dielectric response of each shell is calculated as

$$
\operatorname{Im}\left[\frac{-1}{\varepsilon^{n l}(k, \omega)}\right]=4 \pi \int_{0}^{R_{\mathrm{WS}}} \operatorname{Im}\left[\frac{-1}{\varepsilon\left(k, \omega, k_{n l}^{F}(r), E_{n l}\right)}\right] r^{2} d r,
$$

where $R_{\mathrm{WS}}$ is the atomic Wigner-Seitz radius, $\delta_{n l}(r)$ is the density of electrons, $k_{n l}^{F}(r)=\left[3 \pi^{2} \delta_{n l}(r)\right]^{1 / 3}$ is the local Fermi velocity, and $E_{n l}$ is the ionization threshold for this shell. The local densities and binding energies were obtained from the Hartree-Fock calculations by Clementi and Roetti [26] and Bunge et al. [27].

In the SLPA [3] the ionization threshold is considered explicitly by using the Levine-Louie dielectric function [28] instead of the Lindhard one [29]. It is useful to remark that the Levine-Louie dielectric function satisfies the $f$-sum rule (particle number conservation). This SLPA has also been applied in recent calculations of stopping powers $[3,30]$, energy-loss straggling [31], and multiple-ionization cross sections of rare gases [32].

\section{RESULTS}

The experimental results of the stopping cross section of $\mathrm{Zn}$ for Be and B are plotted in Figs. 4 and 5, respectively. Additionally, the results of Mertens and Krist [6] in the low energy range and the SRIM 2008 values [7] of the electronic stopping power are displayed in these figures, as well as the theoretical calculations showing the separated contribution of the valence and inner-shell electrons.

The theoretical calculations yield a maximum of the stopping cross section for $\mathrm{Be}$ in $\mathrm{Zn}$ at $620 \mathrm{keV} / \mathrm{u}$. For B the energy of the maximum is $670 \mathrm{keV} / \mathrm{u}$. Both values are in very good accordance with the present experimental data.

In the low energy range, the calculations for $\mathrm{Be}$ in $\mathrm{Zn}$ are in good agreement with the experimental data of Mertens and Krist [6]. In the energy range between 60 and $260 \mathrm{keV} / \mathrm{u}$, there are some discrepancies in the stopping cross section, where theory yields lower values than the present experiment. At higher energies the agreement is very good. The SRIM 2008 curve is in good agreement with the present and previous measurements on the whole energy range. However, the position of the maximum predicted by SRIM 2008 is shifted toward lower energies.

For B ions in $\mathrm{Zn}$, the agreements found both with this work and previous experimental data are very good for all

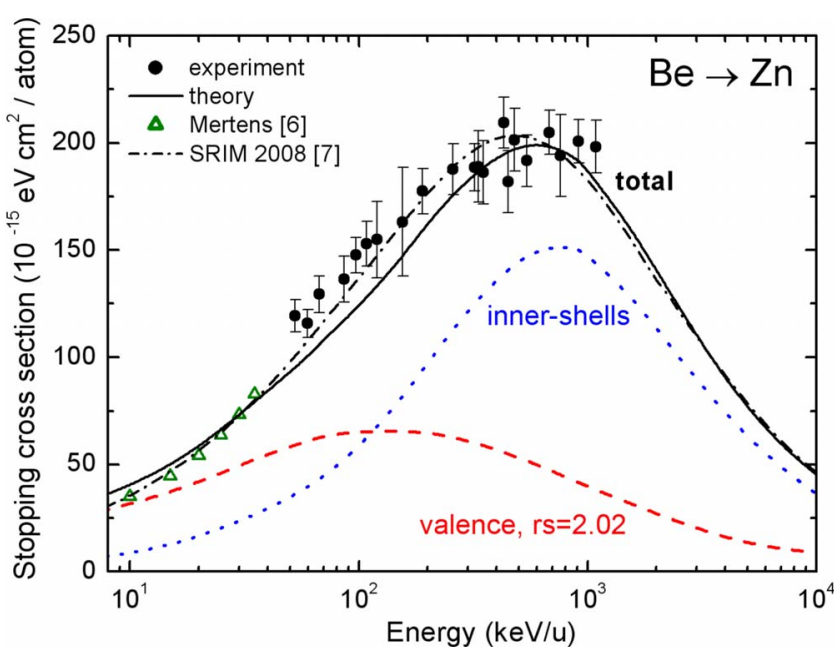

FIG. 4. (Color online) Stopping cross sections of $\mathrm{Be}$ in $\mathrm{Zn}$. Symbols: solid circles, current experimental results; open triangles, Mertens and Krist [6]. Curves: solid line, theory total results; dotted line, contribution of inner shells; dashed line, valence electron contribution; dashed-dotted line, SRIM 2008 values [7].

the energy range, considering the experimental uncertainties. Some minor discrepancies are observed at energies between 150 and $300 \mathrm{keV} / \mathrm{u}$. Additionally, both the experimental and calculated values are in very good agreement with the SRIM 2008 predictions.

It should be noted that a perturbative calculation for the interaction of the projectile with the valence electrons would yield to larger stopping cross-section values (cf. Figs. 2 and 3 ), which shows that a nonlinear approach is required. Also, we note that the theoretical results depend on the projectile charge states, which in this case were taken from the CASP code [25]. In the case of $\mathrm{Be}$ and $\mathrm{B}$ ions in $\mathrm{Zn}$, these values are extrapolated from measurements on other target elements.

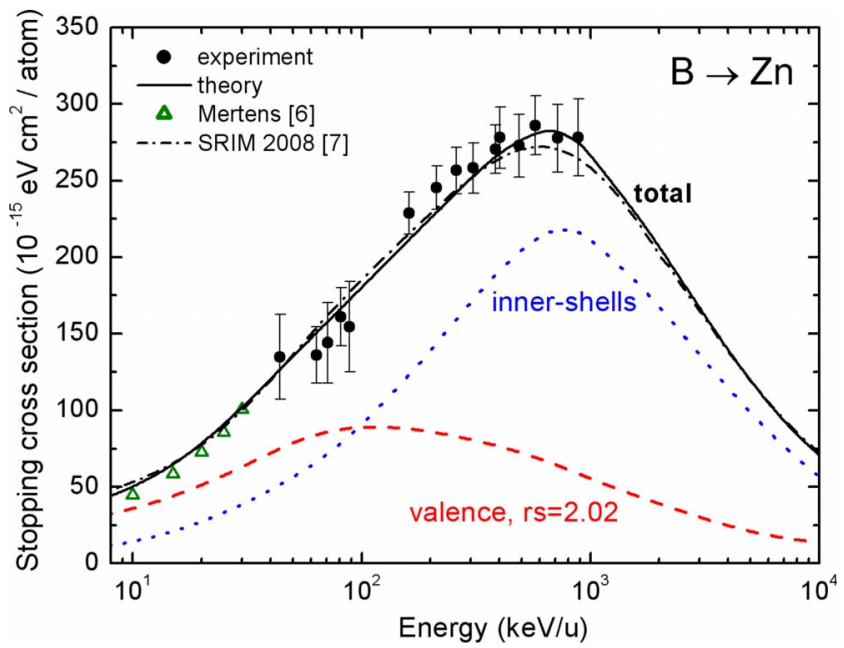

FIG. 5. (Color online) Stopping cross sections of B in Zn. Symbols: solid circles, current experimental results; open triangles, Mertens and Krist [6]. Curves: solid line, theory total results; dotted line, contribution of inner shells; dashed line, valence electron contribution; dashed-dotted line, SRIM 2008 values [7]. 


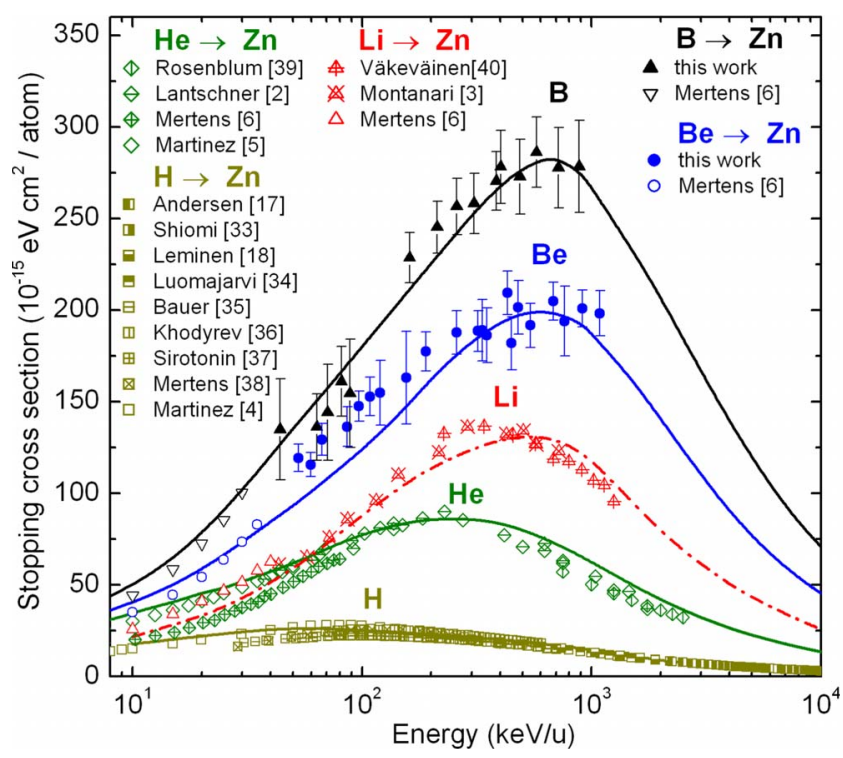

FIG. 6. (Color online) Total stopping of $\mathrm{Zn}$ for $\mathrm{H}, \mathrm{He}, \mathrm{Li}, \mathrm{Be}$, and $\mathrm{B}$ ions. Symbols: experimental data for $\mathrm{H}[4,17,18,33-38], \mathrm{He}$ $[2,5,6,39], \mathrm{Li}[3,6,40], \mathrm{Be}[6]$, and $\mathrm{B}[6]$. Curves present the theoretical calculations of this work.

In Fig. 6 the stopping cross-section data of $\mathrm{Zn}$ for $\mathrm{H}$, He, $\mathrm{Li}, \mathrm{Be}$, and $\mathrm{B}$ are plotted together in order to show a general perspective of the current theoretical developments. A description of the results for $\mathrm{H}$ to $\mathrm{Li}$ was given in a previous work [3]. Taking into account the present measurements and calculations, it is found that the same theoretical scheme is also adequate for heavier ions such as Be and B which involve higher and more charge states. One remarkable characteristic of the present model is that it does not rely on any free parameter or normalization factor.

\section{CONCLUDING REMARKS}

In this work RBS measurements of the stopping power of $\mathrm{Zn}$ for the intermediate mass projectiles $\mathrm{Be}$ and $\mathrm{B}$ are presented in the energy range between $40 \mathrm{keV} / \mathrm{u}$ and $1 \mathrm{MeV} / \mathrm{u}$ together with $a b$ initio calculations. The explored energy region includes the maximum of the stopping power for both ions, which represents a real challenge for a theoretical description.
For both projectiles a good description is obtained with the presented theoretical formulation, which includes the nonperturbative extended Friedel sum rule-transport crosssection formalism for the valence electron contribution and the shellwise local plasma approximation for the target inner shells.

The theoretical scheme calculates the separate contribution of each projectile charge state. The semiempirical predictions of CASP [25] were used to represent the charge state fractions.

The agreement between theory and experiment is satisfactory in both cases (i.e., Be and B), although we note some discrepancies at intermediate energies in the case of $\mathrm{Be}$. We also emphasize the good overall agreement of our results with the SRIM predictions.

We note that in order to achieve a good agreement between theory and experiment, it is essential to use a nonlinear description for the interaction of the projectile with the valence electrons. This framework is particularly useful at low energies, where the contribution of the valence electrons to the stopping power is predominant and screening effects are very important.

The present theoretical approach provides a method to separate the contributions of valence and inner-shell target excitations to the stopping power. The consistency of the theoretical method has been tested by extending the comparison with experiments to the sequence of ions $\mathrm{H}, \mathrm{He}, \mathrm{Li}, \mathrm{Be}$, and $\mathrm{B}$, covering a wide range of energies appropriate to illustrate the transition from low to high energies. The theoretical curves describe fairly well the experimental results for these five elements, with some discrepancies, as previously indicated, in the case of Be. However, for the heavier B ions the good agreement is recovered. Therefore, we think it may be useful to continue this line of research by considering heavier ions. Work along this line is currently in progress.

\section{ACKNOWLEDGMENTS}

Several useful comments by P. Grande are gratefully acknowledged. This work was partially supported by the Universidad de Buenos Aires, the Consejo Nacional de Investigaciones Científicas y Técnicas, the Agencia Nacional de Promoción Científica y Tecnológica from Argentina, and by the CNPQ from Brazil. E.D.C. acknowledges support from Consejo Nacional de Investigaciones Científicas y Técnicas (CONICET), Argentina.
[1] H. Paul, http://www.exphys.uni-linz.ac.at/stopping/

[2] G. H. Lantschner, J. C. Eckardt, A. F. Lifschitz, N. R. Arista, L. L. Araujo, P. F. Duarte, J. H. R. dos Santos, M. Behar, J. F. Dias, P. L. Grande, C. C. Montanari, and J. E. Miraglia, Phys. Rev. A 69, 062903 (2004).

[3] C. C. Montanari, J. E. Miraglia, M. Behar, P. F. Duarte, N. R. Arista, J. C. Eckardt, and G. H. Lantschner, Phys. Rev. A 77, 042901 (2008).

[4] G. Martinez-Tamayo, J. C. Eckardt, G. H. Lantschner, and N.
R. Arista, Phys. Rev. A 51, 2285 (1995).

[5] G. Martinez-Tamayo, J. C. Eckardt, G. H. Lantschner, and N. R. Arista, Phys. Rev. A 54, 3131 (1996).

[6] P. Mertens and Th. Krist, J. Appl. Phys. 53, 7343 (1982).

[7] J. F. Ziegler and J. P. Biersack, SRIM-2008.03-The Stopping and Range of Ions in Matter. Code available in http:// www.srim.org

[8] A. F. Lifschitz and N. R. Arista, Phys. Rev. A 57, 200 (1998).

[9] A. F. Lifschitz and N. R. Arista, Phys. Rev. A 58, 2168 (1998). 
[10] N. R. Arista, Nucl. Instrum. Methods Phys. Res. B 195, 91 (2002).

[11] J. Lindhard, and M. Scharff, K. Dan. Vidensk. Selsk. Mat. Fys. Medd. 27, 15 (1953).

[12] E. Bonderup, K. Dan. Vidensk. Selsk. Mat. Fys. Medd. 35, 17 (1967).

[13] W. K. Chu and D. Powers, Phys. Lett. 40A, 23 (1972).

[14] R. E. Johnson and M. Inokuti, Comments At. Mol. Phys. 14, 19 (1983).

[15] J. D. Fuhr, V. H. Ponce, F. J. Garcia de Abajo, and P. M. Echenique, Phys. Rev. B 57, 9329 (1998).

[16] C. C. Montanari and J. E. Miraglia, Phys. Rev. A 73, 024901 (2006).

[17] H. H. Andersen, C. C. Hanke, H. Simonsen, H. Sörensen, and P. Vajda, Phys. Rev. 175, 389 (1968).

[18] E. Leminen, A. Fontell, and M. Bister, Ann. Acad. Sci. Fenn., Ser. A6 6, 281 (1968).

[19] N. Bohr, K. Dan. Vidensk. Selsk. Mat. Fys. Medd. 18, 8 (1948).

[20] W. K. Chu, J. W. Mayer, and M. A. Nicolet, Backscattering Spectrometry (Academic, New York, 1978).

[21] A. Dalgarno and G. W. Griffing, Proc. R. Soc. London, Ser. A 232, 423 (1955).

[22] C. Reinhold and J. E. Miraglia, J. Phys. B 20, 1069 (1987).

[23] D. Isaacson, New York University Report No. 02698 (National Auxiliary Publication Service, New York, 1975).

[24] N. D. Mermin, Phys. Rev. B 1, 2362 (1970).

[25] G. Schiwietz and P. L. Grande, Nucl. Instrum. Methods Phys.
Res. B 175-177, 125 (2001); CASP version 4.0. Code available at www.hmi.de/people/schiwietz/casp.html

[26] E. Clementi and C. Roetti, At. Data Nucl. Data Tables 14, 177 (1974).

[27] C. F. Bunge, J. A. Barrientos, and A. V. Bunge, At. Data Nucl. Data Tables 53, 113 (1993).

[28] Z. H. Levine and S. G. Louie, Phys. Rev. B 25, 6310 (1982).

[29] J. Lindhard, K. Dan. Vidensk. Selsk. Mat. Fys. Medd. 28, 8 (1954).

[30] A. J. Garcia and J. E. Miraglia, Phys. Rev. A 74, 012902 (2006).

[31] C. C. Montanari, J. E. Miraglia, S. Heredia-Avalos, R. GarciaMolina, and I. Abril, Phys. Rev. A 75, 022903 (2007).

[32] C. D. Archubi, C. C. Montanari, and J. E. Miraglia, J. Phys. B 40, 943 (2007).

[33] N. Shiomi-Tsuda, N. Sakamoto, and R. Ishiwari, Nucl. Instrum. Methods Phys. Res. B 93, 391 (1994).

[34] M. Luomajärvi, Radiat. Eff. 40, 173 (1979).

[35] P. Bauer, F. Kastner, A. Arnau, A. Salin, P. D. Fainstein, V. H. Ponce, and P. M. Echenique, Phys. Rev. Lett. 69, 1137 (1992).

[36] V. A. Khodyrev, V. N. Mizgulin, E. I. Sirotonin, and A. F. Tulinov, Radiat. Eff. 83, 21 (1984).

[37] E. I. Sirotinin, A. F. Tulinov, V. A. Khodyrev, and V. N. Mizgulin, Nucl. Instrum. Methods Phys. Res. B 4, 337 (1984).

[38] P. Mertens and Th. Krist, Nucl. Instrum. Methods Phys. Res. 194, 57 (1982).

[39] S. Rosenblum, Ann. Phys. 10, 408 (1928).

[40] K. Väkeväinen, Nucl. Instrum. Methods Phys. Res. B 122, 187 (1997). 